\title{
The logic of colors in model-theoretical and game-theoretical perspectives ${ }^{1}$
}

\author{
Elena G. Dragalina-Chernaya
}

\begin{abstract}
This paper sketches two approaches to the color exclusion problem provided by model-theoretical and gametheoretical semantics. The case study, modeling the experimentally confirmed perception of 'forbidden' (e.g., reddish green and bluish yellow) colors, is presented as neuropsychological evidence for game-theoretical semantics.
\end{abstract}

Keywords: invariance criterion, permutation invariance, color exclusion problem, binary colors, opponent-processing model, overdefined games, non-strictly competitive games, payoff independence

\section{Invariance Criterion Revisited}

Logical knowledge of reality is possible since logic deals with formal, metaphysically unchanging features of reality. But what does it mean exactly? How does our formal model of reality depend on more or less sophisticated understanding of logicality?

According to Tarski's model-theoretical approach, a concept is logical if and only if 'it is invariant under all possible one-one transformations of the world onto itself' [16, p. 149 ${ }^{2}$. Felix Klein's famous Erlangen Program (1872) proposed the classification of various

${ }^{1}$ This study comprises research findings from the 'Game-theoretical foundations of pragmatics' Project № 12-03-00528a carried out within The Russian Foundation for Humanities Academic Fund Program.

${ }^{2}$ According to Tarski-Sher's criterion, it is better to discuss 'isomorphisms' (or 'bijections') and 'structures' instead of 'permutations' (or 'transformations') and the 'world'. This criterion is historically traced to Lindström's (1966) generalization of Mostowski's approach (1957) (see [15]). 
geometries according to invariants under suitable groups of transformations. Klein pointed out that each geometric field can be characterized by the invariance condition satisfied by its notions. Tarski's criterion of logicality extended this idea to the domain of logic. Permutation invariance takes all one-one transformations into account and as a result, characterizes, according to Tarski, the most general notions. For Tarski, the science which studies these notions is logic. If we interpret the formality of a theory as its invariance under permutations of the universe it means that the theory does not distinguish between individual objects and characterizes only those properties of a model which do not depend on its nonstructural transformations. Formal property should be preserved under the arbitrary switching of individual objects. For example, 'red' and 'green' are non-formal properties, since they distinguish between things which are red and green.

However the standard argument in favor of invariance under permutation, which relies on the generality of logic, may be challenged. Tarski considered the class of permutations as the most general class of nonstructural transformations, since permutations do not respect any extra-structure. On the contrary, as Denis Bonnay pointed out, there are a lot of other concepts of similarity (i.e. approximate preservation) between structures which are far less demanding then Tarski's criterion. Thus, 'even if one grants that generality is a good way to approach logicality, there is no evidence that the class of all permutations is the best applicant for the job' [2, p. 38]. On the other hand, Ludwig Wittgenstein, for example, does not consider generality as a defining attribute of logicality; 'The mark of a logical proposition is not general validity... [18, 6.1231]. The general validity of logic might be called essential, in contrast with the accidental general validity of such propositions as 'All men are mortal' [18, 6.1232]'. Yet, what kind of general validity is essential and, as a result, logical for Wittgenstein?

\section{Invariance Criterion Generalized}

According to Tractatus, it is logically impossible for two colors to be at one place at the same time. This is because of the 'logical structure of color'. As Wittgenstein pointed out, 
'Just as the only necessity that exists is logical necessity, so too the only impossibility that exists is logical impossibility... [18, 6.375]. For example, the simultaneous presence of two colours at the same place in the visual field is impossible, in fact logically impossible, since it is ruled out by the logical structure of colour (It is clear that the logical product of two elementary propositions can neither be a tautology nor a contradiction. The statement that a point in the visual field has two different colours at the same time is a contradiction.)' $[18$, 6.3751].

According to Wittgenstein, color ascriptions should be elementary. But, as the concluding remark implies, they cannot be elementary; the color ascriptions are logically interdependent, and Wittgenstein said that elementary propositions are independent. This is a well-known problem of color exclusion.

In Some Remarks on Logical Form Wittgenstein offered a solution to this problem. Here he is interested in examining what he calls the 'logical structure' or the 'logical form' of the 'phenomena'. As he says, 'we can only arrive at a correct analysis by, what might be called, the logical investigation of the phenomena themselves, i.e., in a certain sense a posteriori, and not by conjecturing about a priori possibilities' [19, p. 163].

A posteriori color-incompatibility claims don't express experience in its usual sense. These tautologies are logically valid due to the geometrical organization of color space. However, unlike Kant, this appeal to geometry does not entail the synthetical character of the corresponding statements. The point is that color space is a 'space of possibilities' which is for Wittgenstein a logical space.

If our logic takes into account a spectrum of invariance which preserves several additional structures, for example, a structure of color space, we may get various types of logical invariance. Therefore, following Wittgenstein we turn back from Tarski's permutation invariance criterion to Klein's original program. From the point of view of Klein's ideology, the logic of colors may be considered as a member of a family of various logics whose notions are invariant for 
one-one transformations which respect additional formal structures, in particular, the formal relations of colors. The invariance criterion which is generalized in this way is wide enough to include not only one extreme type of invariance (i.e. permutation invariance), but a variety of invariances which respect different types of ordering of the universe (see also [17, p. 320]).

\section{Wittgenstein's 'puzzle proposition'; meaning postulates or mapping functions?}

Now the key question is the following: Why did Wittgenstein consider relations between colors as formal, logical ones? My main concern is to clarify Wittgenstein's 'puzzle proposition' from Remarks on Colour that 'there can be a bluish green but not a reddish green'.

In his famous paper Reds, Greens, and Logical Analysis Hilary Putnam pointed out, that Wittgenstein's 'puzzle proposition' is analytic, in the sense in which 'analytic' means 'true on the basis of definitions plus logic'. He proposed to define the second-level predicates ' $\operatorname{Red}(F)$ ' (for ' $F$ is a shade of red') and ' $\operatorname{Grn}(F)$ ' (for ' $F$ is a shade of green'). In defining these predicates we must be restricted, in particular, by the postulate: 'Nothing can be classified as both a shade of red and a shade of green (i.e., 'that shade of red' and 'that shade of green' must never be used as synonyms)' [12, p. 216]. Putnam's approach to color-incompatibility has gained widespread acceptance among recent writers on perception. As Larry Hardin says in Color for Philosophers, 'Perhaps not being red is part of the concept of being green. Yet it seems that all a normal human being has to do to have the concept of green is to experience green in an appropriately reflective manner' [5, p. 122] (see also [22]).

Nevertheless, the introduction of certain meaning postulates seems to be irrelevant to the exegesis of Wittgenstein's ideas. The meaning postulates expand a family of analytic truths by means of dictionary conventions. On the contrary, for Wittgenstein, internal relations of colors are elementary (see, e.g. [20, § 80]). His 'puzzle proposition' is 'in a certain sense a posteriori' and its necessity does not rely on the nature of colors or 'normal human beings', but on the structural relations within the system of colors, i.e. on the geometry of colors. The objective basis for the necessity of the 
color-incompatibility claims is the geometry of color space as 'part of the method of projection by which the reality is projected into our symbolism' [19, p. 166].

Contrary to the meaning postulates approach, Jaakko Hintikka and Merrill Hintikka proposed to represent the concept of color 'by a function $c$ which maps points in visual space into a color space. Then the respective logical forms of 'this patch is red' and 'this patch is green' would be $c(a)=r$ and $c(a)=g$, where $r$ and $g$ are the two separate objects red and green, respectively. The logical incompatibility of the two color ascriptions is then reflected according to Wittgensteinian principles by the fact that the colors red and green are represented by different names. And if so, the two propositions are logically incompatible in the usual logical notation. Their incompatibility is shown by their logical representation: a function cannot have two different values for the same argument because of its 'logical form', i.e., because of its logical type' [6, p. 161]. As Jaakko Hintikka pointed out, 'nonlogical analytical truths sometimes turn out to be logical ones when their structure is analyzed properly' $[8$, p. 52].

Now here is a new puzzling question; is it possible to generalize Hintikka's approach on binary colors, e.g., on reddish green or bluish yellow?

\section{The opponent-processing model of binary colors vision}

We perceive many colors to be binary - purple, for example, as a mixture of blue and red. We may see bluish red, but it seems impossible to experience a color that would be described as a 'reddish green' or a 'bluish yellow'. Thus, certain antagonistic pairs of colors seem not to be combined to form a binary color.

According to the opponent-processing model of colors which goes back to Ewald Hering's opponent process theory (1878), there are different types of retinal photoreceptors with optimal spectral sensitivity to specific wavelengths. Activity in any one type laterally inhibits the activity of neighboring receptors of the same type (e.g., short, middle or long wavelength receptors). Signals from the cones are assumed to be combined in an opposing fashion to produce op- 
posing signals in retinal ganglion cells. This means that the cells are excited by the presentation of a given color and inhibited by presence of its antagonist. Red-green and blue-yellow are supposed to be spectrally opposing channels. Thus, it would be impossible for a human observer to perceive both red and green (blue and yellow) simultaneously, as that percept would require the simultaneous transmission of positive and negative signals in the same channel. As red cancels green and blue cancels yellow, reddish green and bluish yellow are considered to be 'forbidden' binary colors by the opponent-processing model.

The most surprising results in modern neuropsychological literature on color vision are reports that reddish green and yellowish blue colors can be perceived (see, for example, [1] and [3]). In violation of the classical opponent-processing model, 'stabilized-image' experiments have shown that by stabilizing the retinal image between an antagonistic pair of red/green or blue/yellow bipartite equiluminant fields the entire region can be perceived simultaneously as both red and green (blue and yellow) or, to be more precise, as a 'forbidden' homogeneous mixture color whose red and green (blue and yellow) components were as clear as, for example, the green and blue components of aqua.

The first attempt at modeling these opponency violations by Hewitt Crane and Thomas Piantanida was based on the hypothesis that there is an extra stage of cortico-cortical rather then retinocortical visual processing, i.e. a non-opponent filling-in mechanism [3, p. 1079]. The game-theoretical approach allows us to offer a uniform explanation both to standard opponent perception and to its violations in 'stabilized-image' experiments.

\section{5 'Forbidden' binary colors as evidence for game-theoretical semantics}

From the very beginning, the opponent-processing model of colors developed in the game-theoretical framework. It suggested that the basis for color sensations lies in a process of winner-take-all competition between red and green (blue and yellow). Now it is clear that this model must take into account the competitive interactions between teams of color-labeled wavelength-selective cells. As Vincent 
Billock, Gerald Gleason and Brian Tsou pointed out, 'Recent models of cortical color processing suggest that cortical color opponency may not be based on hard-wired wavelength opponency within a single cell but rather on (potentially fragile) interactions between cortical color-sensitive cells' [1, p. 2399]. They assumed that the struggle between red- and green- (blue- and yellow-) labeled units is simply blocked by the border synergy of equilumininance and stabilization [1, p. 2401].

I suppose that there is no need to block the game processing as a whole, as this synergistic effect may be captured by the gametheoretical notion of payoff independence introduced by Ahti-Veikko Pietarinen (see [13]). The main idea of my proposal is the interpretation of opponency violations as payoff independence in 'stabilizedimage' games between red/green or blue/yellow teams of cortical color-sensitive cells. In winner-take-all games, the following holds; if there is a winning strategy of the red team then there does not exist a winning strategy of the green team, and vice versa. In 'stabilizedimage' games the information exchange between the opponent teams is blocked by the synergy of equilumininance and stabilization on the cortical strategic meta-level. Consequently, both red and green (blue and yellow) teams have winning strategies in these games. In other words, 'stabilized-image' games are over-defined. Thus, the law of non-contradiction fails in the generalized logic of colors allowing the simultaneous perception of antagonistic pairs of colors. In contrast to winner-take-all games, 'stabilized-image' games are non-strictly competitive (on over-defined and non-strictly competitive games see papers by Ahti-Veikko Pietarinen and Gabriel Sandu, e.g. [14]).

Evidently, the process of 'negotiations' between teams of opponent colors is nonlinear and gradual. As shown by Billock, Gleason and Tsou, transparency and gradient effects preceded perception of homogeneous 'forbidden' colors. Their experiments also illustrated an entirely novel percept (4 out of 7 subjects) in which the red and green (or blue and yellow) bipartite fields abruptly exchange sides (one subject saw a $90^{\circ}$ reorganization of the bipartite fields) [1, pp. 2398-2399]. These experimental data indirectly 
confirm Wittgenstein's statement about different types of space ${ }^{3}$. Switching effects in 'stabilized-image' experiments lead to simultaneous or serial reorganizations of both visual and color spaces. Whereas Wittgenstein clearly does not think that the science, and particularly neuroscience, is relevant to the resolution of philosophical problems, sometimes neuropsychological experiments influences our colors geometry, which, in turn, constitutes what the colors are. Perhaps, tomorrow the invention of special glasses with a built-in eye tracker will make reddish green and bluish yellow common colors of our 'form of life'.

In conclusion, the basic advantage of the game-theoretical approach to the logic of colors is its procedural character. Concerning the logic of binary colors, game-theoretical models seem to be the best, since a variety of game-theoretical independences provides important insights into the theory of opponent-processing. Gametheoretical notion of strategy allows us to generalize Hintikka's approach to colors as mapping functions on binary colors, in particular, on 'forbidden' binary colors.

\section{References}

[1] Billock, V. A., Gleason, G. A., and B. H. Tsou, Perception of forbidden colors in retinally stabilized equiluminant images: an indication of softwired cortical color opponency? Journal of Optical Society of America 18(10):2398-2403, 2001.

[2] Bonnay, D., Logicality and Invariance, Bulletin of Symbolic Logic 14(1):29-68, 2008.

[3] Crane, H. D., and T. P. Piantanida, On seeing reddish green and yellowish blue, Science 221:1078-1080, 1983.

[4] Dragalina-Chernaya, E., Model-Theoretic Languages as Formal Ontologies, in Munz V.A. et al. (eds.), Language and World. Contributions of the Austrian Ludwig Wittgenstein Society, vol. XVII, Kirchberg, 2009, pp. 78-80.

\footnotetext{
${ }^{3}$ For instance, in Philosophical Remarks he refers to color space ( $\S 1$ ), auditive space $(\S 42)$, tactile space $(\S 214)$, pain space $(\S 82)$, visual space (§ 206), kinaesthetic space (§ 73), a space to which both memory and reality belong, the spaces of movement $(\S 140)$, of orientation $(\S 207)$ and the dark-light space $(\S$ 45) (see [11, p. 79]).
} 
[5] Hardin, L., Color for Philosophers, Hackett, Indianapolis and Cambridge MA, 1988.

[6] Hintikka, J., and M. B. Hintikka, Some Remarks on (Wittgensteinian) Logical Form, Synthese 56(2):155-170, 1983.

[7] Hintikka, J., and G. SAndu, Informational Independence as a Semantical Phenomenon, in Fenstad J.E. et al. (eds.), Logic, Methodology and Philosophy of Science, vol. 8, Amsterdam: Elsevier, 1989. P. 571-589.

[8] Hintikka, J., Logical Versus Nonlogical Concepts: An Untenable Dualism? in Rahman S. et al. (eds.), Logic, Epistemology and the Unity of Science, Springer, 2009, pp. 51-56.

[9] Horst, S., Modeling, Localization and the Explanation of Phenomenal Properties: Philosophy and the Cognitive Sciences at the Beginning of the Millennium, Synthese 147(3):477-513, 2005.

[10] Clark, A., Qualia and the Psychophysiological Explanation of Color Perception, Synthese 65(3):377-405, 1985.

[11] Mulligan, K., Colors, Corners and Complexity; Mejnong \& Wittgenstein on some Internal Relations, in B. C. van Fraassen et al. (eds.), Existence and Explanation: Essays in Honor of Karel Lambert, The University of Western Ontario Series in Philosophy of Science Dordrecht: Kluwer, 1991, pp. 77-101.

[12] Putnam, H., Reds, Greens, and Logical Analysis, The Philosophical Review 65(2):206-217, 1956.

[13] Pietarinen, A.-V., Independence-Friendly Logic and Games of Information, in J. van Benthem et al. (eds.), The Age of Alternative Logics: Assessing Philosophy of Logic and Mathematics Today, Springer, 2006, pp. 243-259.

[14] Pietarinen, A.-V., and G. Sandu, IF Logic, Game-Theoretical Semantics, and the Philosophy of Science, in Rahman S. et al. (eds.), Logic, Epistemology and the Unity of Science, Springer, 2009, pp. 105138.

[15] Sher, G., The Bounds of Logic: A Generalized Viewpoint, Cambridge: MIT, 1991.

[16] TARski, A., What are Logical Notions? History and Philosophy of Logic 7:143-154, 1986.

[17] Van Benthem, J., Logical Constants Across Varying Types, Notre Dame Journal of Formal Logic 30:315-342, 1989.

[18] Wittgenstein, L., Tractatus Logico-Philosophicus, London: Routledge \& Kegan Paul., 1922. 
[19] Wittgenstein, L., Some Remarks on Logical Form, Proceedings of the Aristotelian Society 9:162-171, 1929.

[20] Wittgenstein, L., Philosophical Remarks, Oxford: Blackwell, 1964.

[21] Wittgenstein, L., Remarks on Colour, Oxford: Blackwell, 1977.

[22] Westphal, J., Conflicting Appearances, Necessity and the Irreducibility of Propositions about Colours, Proceedings of the Aristotelian Society, New Series 105:219-235, 2005. 